\title{
Biomechanical assessment and 3D finite element analysis of the treatment of tibial fractures using minimally invasive percutaneous plates
}

\author{
XIN-JIA HU and HUA WANG \\ Department of Bone and Joint Surgery, Shenzhen People's Hospital, 2nd Clinical Medical College of Jinan University, \\ Shenzen, Guangdong 518020, P.R. China
}

Received December 18, 2014; Accepted December 15, 2016

DOI: $10.3892 / e t m .2017 .4629$

\begin{abstract}
The aim of the present study was to investigate the biomechanical effects of varying the length of a limited contact-dynamic compression plate (LC-DCP) and the number and position of screws on middle tibial fractures, and to provide biomechanical evidence regarding minimally invasive plate osteosynthesis (MIPO). For biomechanical testing, 60 tibias from cadavers (age at mortality, 20-40 years) were used to create middle and diagonal fracture models without defects. Tibias were randomly grouped and analyzed by biomechanic and three-dimensional (3D) finite element analysis. The differences among LC-DCPs of different lengths (6-, 10- and 14-hole) with 6 screws, 14-hole LC-DCPs with different numbers of screws (6, 10 and 14), and 14-hole LC-DCPs with 6 screws at different positions with regard to mechanical characteristics, including compressing, torsion and bending, were examined. The 6-hole LC-DCP had greater vertical compression strain compared with the 10- and 14-hole LC-DCPs $(\mathrm{P}<0.01)$, and the 14-hole LC-DCP had greater lateral strain than the 6-and 10-hole LC-DCPs $(\mathrm{P}<0.01)$. Furthermore, significant differences in torque were observed among the LC-DPs of different lengths $(\mathrm{P}<0.01)$. For 14 -hole LC-DCPs with different numbers of screws, no significant differences in vertical strain, lateral strain or torque were detected $(\mathrm{P}>0.05)$. However, plates with 14 screws had greater vertical strain compared with those fixed with 6 or 10 screws $(\mathrm{P}<0.01)$. For 4 -hole LC-DCPs with screws at different positions, vertical compression strain values were lowest for plates with screws at positions $1,4,7,8,11$ and 14 $(\mathrm{P}<0.01)$. The lateral strain values and vertical strain values for plates with screws at positions $1,3,6,9,12$ and 14 were
\end{abstract}

Correspondence to: Dr Hua Wang, Department of Bone and Joint Surgery, Shenzhen People's Hospital, 2nd Clinical Medical College of Jinan University, 1017 Dongmen North Road, Shenzhen, Guangdong 518020, P.R. China

E-mail: wangh_wh@126.com

Key words: tibial fractures, internal fixation, biomechanics, finite element analysis significantly lower compared with those at the other positions $(\mathrm{P}<0.01)$, and torque values were also low. Thus, the 14-hole LC-DCP was the most stable against vertical compression, torsion and bending, and the 6-hole LC-DCP was the least stable. However, the use of 14 screws with a 14-hole LC-DCP provided less stability against bending than did 6 or 10 screws. Furthermore, fixation with distributed screws, in which some screws were close to the fracture line, provided good stability against compression and torsion, while fixation with screws at the ends of the LC-DCP provided poor stability against bending, compressing and torsion.

\section{Introduction}

Minimally invasive plate osteosynthesis (MIPO) has been broadly applied clinically following the improved understanding of the blood supply and local biological environment of fractures. With the development of clinical technology in China, MIPO technology has gradually matured. It is a new development for internal fixation using biological osteosynthesis (BO), in which direct exposure of the fracture site is not necessary. MIPO involves indirect reduction and fixation, which minimizes regional damage to soft tissues and the blood supply of the periosteum, as well as the incidence of nonunion and infection, and so is beneficial with regard to functional rehabilitation and clinical therapeutic effects (1).

The principle of MIPO is biological fixation at the near- and far-ends of the fracture site $(2,3)$. It has a number of advantages compared with traditional open reduction and internal fixation, and intramedullary nail fixation. Firstly, it has a relatively short surgery time $(4,5)$. Secondly, it effectively avoids complications $(6,7)$. Thirdly, it avoids nerve damage and reduces scarring. Fourthly, it exhibits strong healing effects $(8,9)$. Fifthly, it effectively reduces damage of the soft tissues and protects the blood supply of the fracture site (10). Finally, it is able to endure large deformation forces (11).

However, there are many contentious aspects of MIPO application in the treatment of tibial fractures, particularly the length of the limited contact-dynamic compression plate (LC-DCP), the number of screws and the fixing positions, and there are no clear conclusions with regard to the optimum parameters. 
Therefore, in the present study 60 tibias from cadavers were used for the examination of tibial fractures by biomechanical and finite element analysis, in order to provide evidence regarding the treatment of middle tibial fractures using MIPO with $\mathrm{BO}$.

\section{Materials and methods}

\section{Materials}

Biomechanical testing. Wet tibial samples $(n=60)$ were collected from cadavers from individuals with an age at mortality of 20-40 years at the Department of Human Anatomy of Xiangya School of Medicine, Central South University (Changsha, China). Ethical approval was obtained from the institution. The tibias were inspected and X-ray examination was performed, in order to exclude damage and bone pathology, prior to storage at $-20^{\circ} \mathrm{C}$. The fixation materials, namely 6-hole LC-DCPs (model 4.5; length, $105 \mathrm{~mm}$, width, $8 \mathrm{~mm}$; thickness, $3.5 \mathrm{~mm}$ ), 10-hole LC-DCPs (model 4.5; length, $178 \mathrm{~mm}$; width, $8 \mathrm{~mm}$; thickness, $3.5 \mathrm{~mm}$ ), 14-hole LC-DCPs (model 4.5; length, $250 \mathrm{~mm}$; width, $8 \mathrm{~mm}$; thickness, $3.5 \mathrm{~mm})$, cortical stainless steel bone screws $(\Phi, 4.5 \mathrm{~mm})$, and $\mathrm{AO}$ internal fixing equipment were obtained from Bangli Trading Co. (Yangjiang, China).

$3 D$ finite element analysis. Five tibias $30-38 \mathrm{~cm}$ in length (mean, $34 \mathrm{~cm}$ ) were collected from the Department of Human Anatomy of Xiangya School of Medicine, Central South University. The other materials used were the same as those described above.

\section{Analytical instrumentation}

Biomechanical testing. The MTS 858 Mini Bionix universal biomechanics testing system (MTS Systems Corporation, Eden Prairie, MN, USA) was supported by the Department of Human Anatomy of Southern Medical University.

Three-dimensional (3D) finite element analysis. Analysis was conducted using spiral computed tomography (CT), a computer, AutoCAD software (Autodesk, Inc., San Rafael, CA, USA) and finite element software Ansys 6.5.

Biomechanical testing methods. Middle and diagonal fracture models without defects were established using the collected tibias. The tibias were randomly grouped and analyzed by biomechanics and 3D finite element methods. An analysis and comparison of LC-DCPs with different lengths (6-, 10- and 14-hole) combined with 6 screws, 14-hole LC-DCPs combined with different numbers of screws (6,10 and 14), and 14-hole LC-DCPs with 6 screws fixed at different positions was conducted. The positions fixed were as follows (from top to bottom): Group 1, holes 1, 2, 7, 8, 13 and 14; group 2, holes 1, 4, 7, 8, 11 and 14; group 3, holes 1, 3, 6, 9, 12 and 14; and group 4, holes 1-3 and 12-14. Mechanical characteristics, including compression, torsion and 3-point bending, respectively, were evaluated. The load of vertical compression was 0-1,000 N, the torsion angle was $0-3^{\circ}$, and the load of 3 -point bending was $0-400 \mathrm{~N}$. The strain values on torsion, and with 3-point bending and compression were measured and analyzed.

$3 D$ reconstruction of tibial stem finite element models. The tibias were scanned using spiral CT and continuous cross-sectional images $1 \mathrm{~mm}$ in thickness were obtained. The images were analyzed as lattice images and digitized. Using a graphic boundary automatic recording program, created by the Laboratory of Human Anatomy and Biomechanics of Southern Medical University (China), the boundary conditions of the images were recorded as $2 \mathrm{D}$ coordinates in all levels of the tibial images according to the requirements for $3 \mathrm{D}$ reconstruction. With reference to the plane distance and inter-lamellar space, the 2D coordinates were converted into $3 \mathrm{D}$ coordinates following regulation to a suitable spatial scale.

The mechanical values of the tibial materials were searched and switched using the AutoCAD modeling software. The middle tibial fracture model was established with internal fixation using 3D Max.

Using the finite element software Ansys 6.5, the 3D tibial spatial model was established according to modeling principles on points, lines, regions and bodies from top to bottom. CT scanning was conducted in cross-sectional directions in parallel with the xy plane, with the longitudinal direction along the $\mathrm{z}$-axis.

In the digital tibia models, there were 265 reconstruction levels in total. The $\mathrm{z}$-axis was established according to the actual height. The corresponding junctions were connected in each level and geometric models of the tibias were formed. Different colors were used to distinguish different levels and structures, and the element was then divided into different gridding.

\section{$3 D$ reconstruction of $M I P O$-treated middle tibia fractures}

Establishing middle tibial fractures with internal fixation. Five tibias were used to create middle and diagonal fracture models without defects. They were tilted $45^{\circ}$ from the tibial eminence backwards, with an average slope length of $35 \mathrm{~mm}$. A 14-hole LC-DCP and 6 screws were used for fixation, with the fracture line at the middle of the plate. From top to bottom, the fixing positions were as follows: Tibia-1 model, 1st, 2nd, 3 rd, 12th, 13th and 14th holes; tibia-2 model, 1st, 4th, 7th, 8th, 11th and 14th holes; tibia-3 model, 1st, 3rd, 6th, 9th, 12th and 14th holes; tibia-4 model, 1st, 2nd, 7th, 8th, 13th and 14th holes. In the tibia-5 model, all 14 holes were fixed with screws.

$3 D$ reconstruction of tibial fractures with internal fixation. The fixed tibias were scanned by spiral CT and continuous cross-sectional images of $1 \mathrm{~mm}$ thickness were obtained. The images were analyzed as lattice images, digitized and used to establish digital tibia models with 265 reconstruction levels as described above.

Loading the digital model of middle tibia fracture internal fixation. The tibia-1 model with fixation positions at the 1st, 2nd, 3rd, 12th, 13th and 14th holes was loaded. i) Fixation of the digital model was simulated. According to the anatomical structure and biomechanical characteristics of the tibia, a 14-hole plate and 6 screws were used for digital tibia model fixation. ii) Axial compression of the digital model was detected. Following fixation of the digital middle tibial fracture model with a 14-hole plate and 6 screws, the LC-DCP was compressed with a load of $0-1,000 \mathrm{~N}$ vertically and evenly. Data were collected and the vertical and lateral strain values were obtained. Data were analyzed with the load of 1,000 N. iii) Torsion of the digital model was detected. Following fixation of the digital middle tibial fracture model with a 14-hole 
plate and 6 screws, torsional stiffness of the left and right directions was detected using a torsion rate of $2 \% \mathrm{sec}$ and angle of $3^{\circ}$. The torque was obtained from the collecting system and analyzed statistically.

The tibia-2, -3, - 4 and -5 models were each loaded and analyzed according to the procedure described above.

Statistical analysis. All data were collected automatically and analyzed using SPSS 12.0 software (SPSS, Inc., Chicago, IL, USA). Analysis of variance was employed for pairwise comparisons, followed by Tukey's post hoc testing. $\mathrm{P}<0.05$ was considered to indicate a statistically significant difference.

\section{Results}

Biomechanical effects of LC-DCP length on middle tibial fractures. For vertical compression, the strain values for 6-, 10 - and 14-hole LC-DCPs were 0.449 $\pm 0.241,0.093 \pm 0.003$ and $0.139 \pm 0.005$, respectively. The strain values for the 10- and 14-hole plates were significantly lower than those for the 6-hole plate $(\mathrm{P}<0.01)$. The lateral strain values for $6-$, 10- and 14-hole LC-DCPs were $0.120 \pm 0.000,0.1275 \pm 0.010$ and $0.237 \pm 0.000$, respectively. The lateral strain values for the 6- and 10-hole LC-DCPs were significantly different from those for the 14-hole LC-DCP $(\mathrm{P}<0.01)$; however, no significant difference was identified between 6- and 10-hole LC-DCPs $(\mathrm{P}>0.05)$. The torques of the 6-, 10- and 14-hole LC-DCPs were $5.066 \times 10^{-3} \pm 2.715 \times 10^{-3}, 5.671 \times 10^{-3} \pm 2.527 \times 10^{-3}$ and $4.570 \times 10^{-3} \pm 2.228 \times 10^{-3} \mathrm{~N} \cdot \mathrm{m}$, and the vertical strain values of 3 -point bending were $0.049 \pm 0.009,0.124 \pm 0.017$ and $0.062 \pm 0.009$, respectively. Pairwise comparisons for LC-DCPs of different lengths identified significant differences $(\mathrm{P}<0.05)$.

Biomechanical effects of screw number on middle tibial fractures. For vertical compression using 14-hole LC-DCPs, the strain values for 6,10 and 14 screws were $0.120 \pm 0.006$, $0.111 \pm 0.012$ and $0.116 \pm 0.008$, respectively, with no significant difference revealed by pairwise comparisons $(\mathrm{P}>0.05)$. The lateral strain values for 6,10 and 14 screws were $0.234 \pm 0.007$, $0.234 \pm 0.000$ and $0.179 \pm 0.002$, respectively, without significant differences revealed by pairwise comparisons $(\mathrm{P}>0.05)$. The torques for 6,10 and 14 screws were $7.304 \times 10^{-3} \pm 3.072 \times 10^{-3}$, $6.069 \times 10^{-3} \pm 3.086 \times 10^{-3}$ and $7.1295 \times 10^{-3} \pm 3.908 \times 10^{-3} \mathrm{~N} \cdot \mathrm{m}$, respectively, without significant differences indicated by pairwise comparisons $(\mathrm{P}>0.05)$. The vertical strain values of 3 -point bending for 6,10 and 14 screws were $0.149 \pm 0.098$, $0.139 \pm 0.003$ and $0.258 \pm 0.001$, respectively; the vertical strain values for 6 and 10 screws were was significantly different from those for 14 screws $(\mathrm{P}<0.01)$, while no significant difference was identified between 6 and 10 screws $(P>0.05)$.

Biomechanical effects of fixing positions on middle tibial fractures. For groups 1-4, the strain values of vertical compression were $0.120 \pm 0.006,0.033 \pm 0.003,0.139 \pm 0.005$ and $0.116 \pm 0.008$, respectively, with significant differences between group 2 and the other groups $(\mathrm{P}<0.01)$. The lateral strain values in the four groups were $0.234 \pm 0.007,0.237 \pm 0.0006$, $0.187 \pm 0.002$ and $0.217 \pm 0.002$, respectively, with significant differences between group 3 and the other groups $(\mathrm{P}<0.01)$. The torques were $7.304 \times 10^{-3} \pm 3.072 \times 10^{-3}, 6.965 \times 10^{-3} \pm 3.096 \times 10^{-3}$,
$4.570 \times 10^{-3} \pm 2.228 \times 10^{-3}$ and $2.086 \times 10^{-3} \pm 0.998^{*} 10^{-3} \mathrm{~N} \cdot \mathrm{m}$, respectively, with significant differences identified between group 3 and the other groups $(\mathrm{P}<0.01)$. The strain values of 3 -point bending in the four groups were $0.149 \pm 0.098,0.184 \pm 0.002$, $0.004 \pm 0.001$ and $0.282 \pm 0.000$, respectively. Values for groups 3 and 4 were indicated to be significantly different compared with group 1 or 2 by pairwise comparison $(\mathrm{P}<0.01)$. Group 4 was indicated to be significantly different from the other groups by pairwise comparisons $(\mathrm{P}<0.01)$. However, there was no significant difference between groups 1 and $2(\mathrm{P}>0.05)$.

Stress sustained in the tibia-1 model. For axial compression, the compression and torsion at the 3rd and 12th holes on the $\mathrm{x}$ - and $\mathrm{y}$-axes were the greatest, and accumulated on the fracture. There was no significant difference in the stress on the z-axis among the various screw positions. Thus, the stress on any individual screws was minimized. The main strain was focused on the positions without fixation (Fig. 1).

Stress sustained in the tibia-2 model. For axial compression, the stress on the 1 st hole on the $\mathrm{x}-, \mathrm{y}$ - and $\mathrm{z}$-axes was the greatest, followed by the 4th hole. The stress decreased gradually from the top to the bottom of the plate. With regard to torque, the stress on the 7th and 8th holes on the $\mathrm{x}-, \mathrm{y}$ - and z-axes was the greatest, and the stress tended to decrease gradually from the middle of the fracture to the end. The stress was mainly focused on the center of the plate (Fig. 2).

Stress sustained in the tibia-3 model. For axial compression, the stress on the 1st hole on the $\mathrm{x}-, \mathrm{y}$ - and z-axes was the greatest, followed by the 3 rd hole. The stress decreased gradually from the top to the bottom of the plate. For torque, the stress on the 6th and 9th holes on the $\mathrm{x}-$,, $\mathrm{y}$ - and $\mathrm{z}$-axes was the greatest, with the greater strain on the center without fixation (Fig. 3).

Stress sustained in the tibia-4 model. For axial compression, the stress on the 1 st hole on the $\mathrm{x}-, \mathrm{y}$ - and $\mathrm{z}$-axes was the greatest. The stress decreased gradually from the top to the bottom of the plate. With regard to torque, the stress on the 7 th and 8th holes on the $\mathrm{x}-, \mathrm{y}-$ and $\mathrm{z}$-axes was the greatest, but less than in the tibia-3 model, with a balanced strain distribution (Fig. 4).

Stress sustained in the tibia-5 model. For axial compression, the stress on the 1st hole on the $\mathrm{x}$-, $\mathrm{y}$ and $\mathrm{z}$-axes was the greatest, followed by the 2 nd hole. The stress decreased gradually from the top to the bottom of the plate. For torque, the stress on the 7 th and 8th holes on the $\mathrm{x}-, \mathrm{y}-$ and $\mathrm{z}$-axes was the greatest. The strain was particularly focused on the two screws in the center of the LC-DCP (Fig. 5).

\section{Discussion}

As increases in the energy or speed of damage have occurred, for example, in traffic accidents, the incidence of tibial fractures has notably increased; such fractures account for $10 \%$ of long bone fractures (12). Tibial fractures are predominantly middle fractures (13-17). Therefore, further investigations focused on middle tibial fractures are warranted. 
A
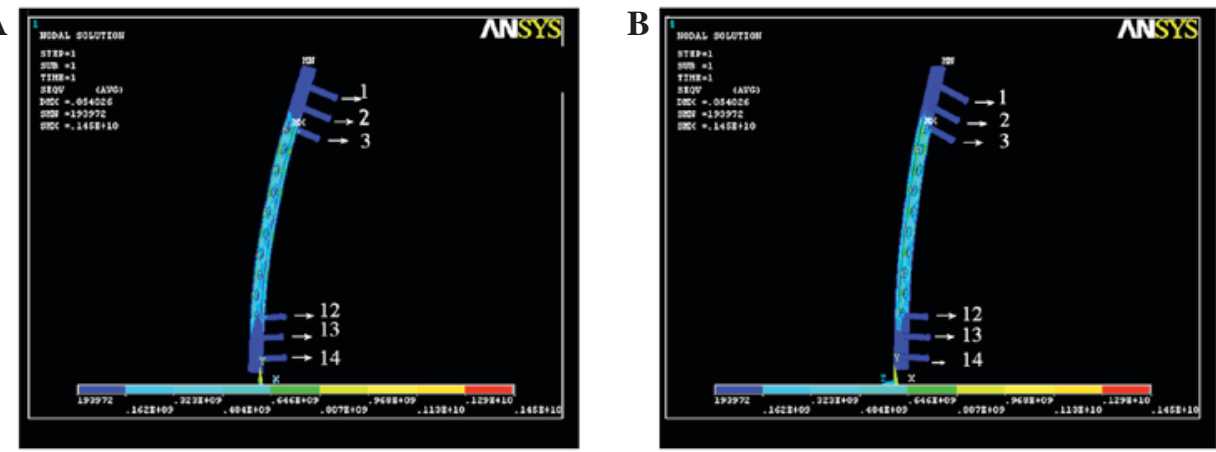

Figure 1. Stress distribution of (A) axial compression and (B) torsion in each shaft torsion at holes 1-3 and 12-14.

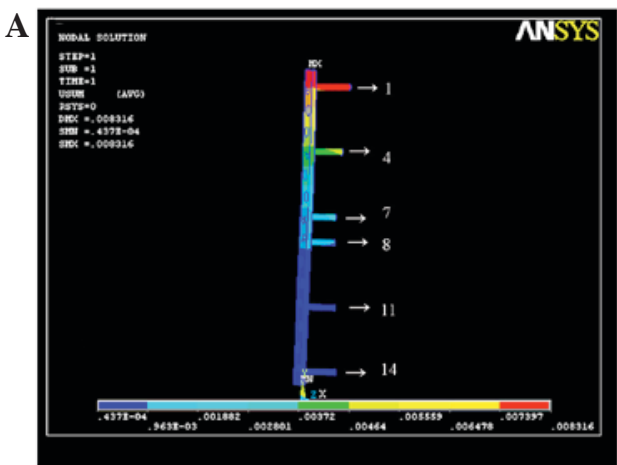

$\mathbf{B}$

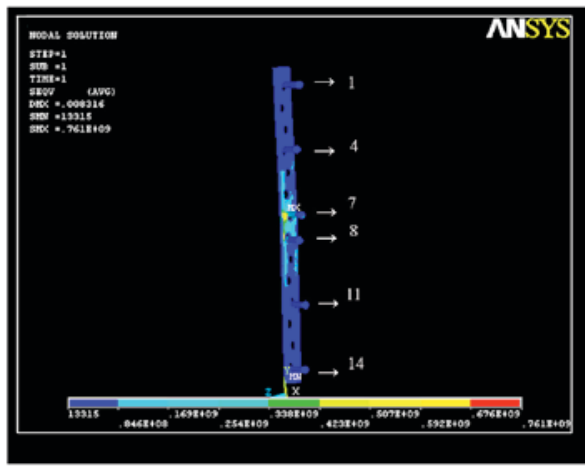

Figure 2. Stress distribution of (A) axial compression and (B) torsion in each shaft torsion at holes 1, 4, 7, 8, 11 and 14

On the basis of the BO concept, investigations on MIPO in the treatment of fractures have been performed and therapeutic effects obtained. MIPO is a novel development for the treatment of fractures, and has been conducted as follows: For indirect reduction, a small incision was opened distally to the fracture; a subcutaneous tunnel was established and the LC-DCP was embedded with fixation $(18,19)$. For broken ends of fractured bone without exposure, the integrity of the soft tissue between the broken ends of fracture bone was maintained thoroughly (20). The integration of soft tissue around the fractured bone provides the healing with beneficial biotic environment as an important co-factor for correcting and sustaining the bone after fixation.

Numerous models have been used in orthopedic biomechanics research, such as animal models, physical models, cadaveric models and finite element analysis. However, there are certain limitations for each kind of model (21-23). Cadaveric models are advantageous in geometrical structure and material characteristics, and are the most appropriate for providing values on biomechanical properties, which are used in surgery for evaluation; however, they lack certain biological features, have higher experimental expense, may be difficult to acquire and have low potential for repeated use, limiting their application $(24,25)$. Finite element analysis is a repeatable and sustainable method of research, and is a relatively novel method for studying orthopedic biomechanics. Finite element analysis is able to reflect interactions regionally and internally, with changes in subjective and material structures, in order to provide a biomechanical evaluation and prognosis for different kinds of disease and injury, fixture equipment and surgical methods $(26,27)$. Finite element analysis in the biomechanical simulation of internal fracture fixation has been widely used, in China and elsewhere. However, due to the complexity of finite element analysis, particularly establishment of the model, its application in orthopedic biomechanics is limited, with confirmation between the results and the practical experiments being necessary. Therefore, in the present study, biomechanics combined with finite element analysis was used to mutually confirm the results of MIPO in the treatment of tibial fractures, in order to provide theoretical evidence for clinical treatment.

He et al (28) studied the effects of screw number on the load of internally fixed structures using a torsion test. Combining their observations with those reported in the literature, they summarized that omitting $40 \%$ of the screws of an LC-DCP had no significant effect on the stiffness for one plate with internal fixation. Therefore, in the present study, two positions at the ends of the LC-DCP were fixed, in order to exclude the effect of length on internal stability.

In the analysis of screw breakage in steel plates used to treat middle long bone fractures, Liu et al (29) found that in addition to anatomical restoration, stability was also critical in internal fixation. It was considered that the stability of fractures was associated with the length of the fixing material, which demanded that the plate length was 5 times as long as the longest fracture line and $\geq 4$ cortices were fixed at one side of an ulnoradial fracture, 6-8 cortices of a humeral fracture, and 8-10 cortices of the tibial or femoral fracture. However, according to the study by Laurence et al (19), 4 screws were sufficient for fixation of one plate with mechanical stability. 

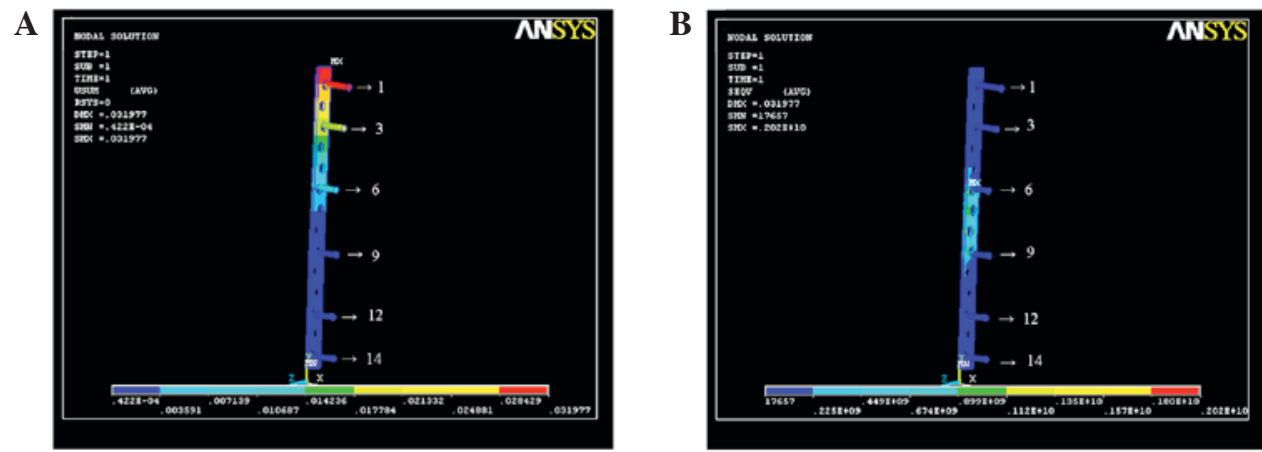

Figure 3. Stress distribution of (A) axial compression and (B) torsion in each shaft torsion at holes 1, 3, 6, 9, 12 and 14 .

A

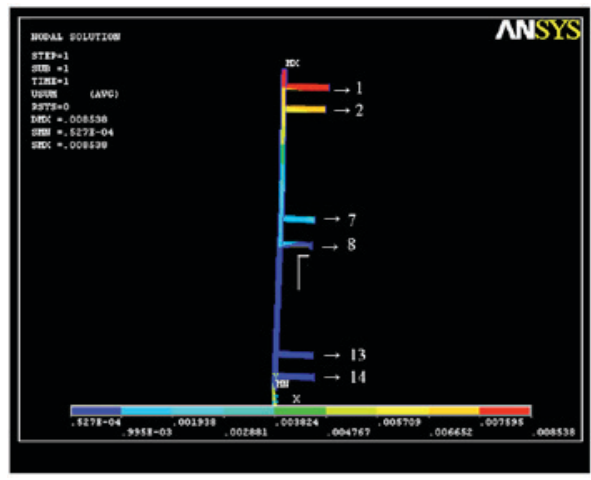

B

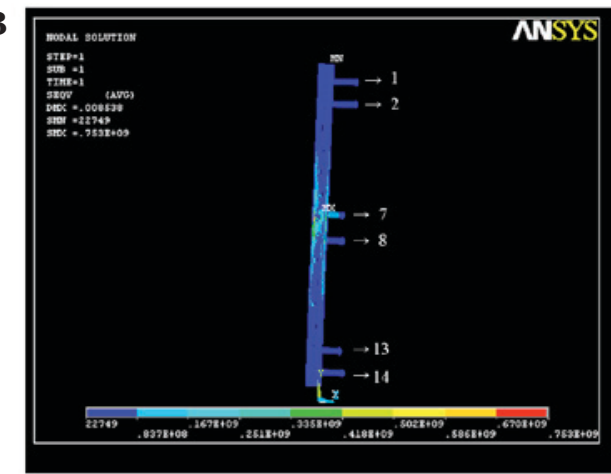

Figure 4. Stress distribution of (A) axial compression and (B) torsion in each shaft torsion at holes 1, 2, 7, 8, 13 and 14
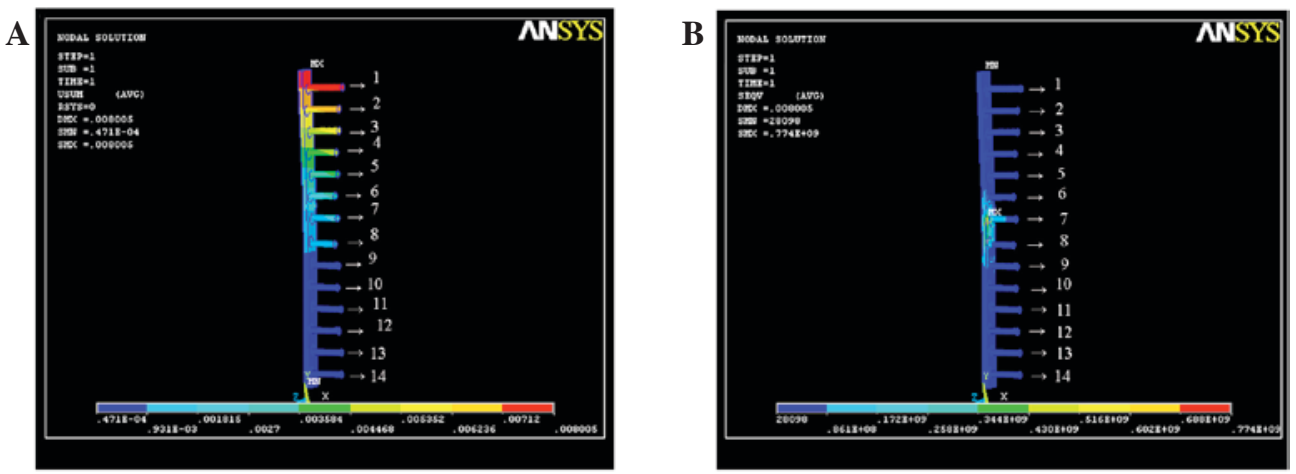

Figure 5 Stress distribution of (A) axial compression and (B) torsion of each nail fixed and loading model for 14 screws in total

Rozbruch et al (20) recommended that the screws should be fixed at the most appropriate position, and for one fracture fragment, there should be 2 or 3 screws for fixation, where the most appropriate position for fixation should enhance the connection between the steel plate and the fracture site, to provide a stable leverage effect. Thus, a 3-point and 6-screw method, which involved fixing the ends of the steel plate with 2 screws and fixing the ends close to the fracture line with 1 screw, could form lever balance fixation with 3 points. On the basis of the analysis described above, a 14-hole LC-DCP was used for the tibial fracture test in the present study. The long plate should be able to correct rotation and angling deformities, and sustain the tibial axis and length. By lengthening the plate, for example, by prolonging the lever arm for increasing the strain distribution instead of focusing on one position, the plate, screws, bone and soft tissues could be combined into a mechanical structure with greater stability.

The results of the present study showed that with the same number of screws, increasing the plate length increased the fixing stability. By analysis of the biomechanical stability, it was identified that a long plate was better than a short one. With the same plate length (14-hole LC-DCP), the stability against bending was decreased as the screw number was increased to 14 , but it was unclear whether an excessive number of screws leads to a reduction of overall stability. Field et al (30) found there was no significant difference in the stability of the internal fixation structure between a steel plate in which some holes had no screws and the same steel plate in which all holes had screws. It has been suggested that increasing the span between the 2 screws at the ends of the plate contributed 
to higher stability than a plate with shorter span and more screws (31). A longer plate could eliminate the use of $40 \%$ of the screws, and increased surface tension and bone healing promotion effects were obtained without effect on fixation. Decreasing the number of screws may reduce skeletal trauma. More importantly, increasing the span between screws may neutralize the external instant stress on the screws when they are extracted.

Ellis et al (27) found that for plates of the same length, fixation with screws at the ends of the plate increased the stiffness against torsion. Another previous study (32) showed that with the same plate length and number of screws, the stability against bending was improved by distributing the screws evenly, while it was reduced by distributing the screws at the ends of the plate. In the present study, a plate with the same material, length, width and number of screws was used, and the fixing position was changed. The vertical compression test showed that with the same plate length and screw number, the stability against compression with fixation close to the fracture line was optimum. The torsion test showed that with the same plate length and screw number, the stability against torsion with fixation close to the fracture line or with even distribution was better that than when fixing screws were distributed at the ends of the plate.

Finite element analysis is able to break up the whole fracture and fixation system into parts, and combine the parts into a whole. The basic principles are as follows: Breaking up the bone sample continuous elastomer into finite elements; connecting the elements with junctions and evaluating the combination instead of the bone sample primary elastomer. The unknown values of a point in the element are analyzed through differences in the functional relationship selected by the number of junctions. In the analysis of the element, for the simple element shape, complex formulas between the junctions are established. The formulas are combined into a general formula and the boundary conditions added. The characteristics of each element and the whole can then be investigated. The more compact the element distribution, the more accurate the calculation, and the higher the quantity of work (21-23).

In the present study, the tibias were scanned by CT and data were collected. Therefore, the models used actual geometry to create ideal models. The 3D tibial fracture model was reconstructed based on the direct measurements made from images of the real sample obtained by spiral CT scanning. The reconstructed models were vivid and objective, were able to reflect the real geometry and biomechanical characteristics of the tibia, and enabled the application of finite element analysis for biomechanical study of the tibia. In this study, we compensated for the deficiency of the finite element analysis on lower body mechanics, and supplied new basic models and ideas to analyze the mechanical characteristics of tibias.

A complete 3D tibial model was constructed, which was collected from scanning images $1 \mathrm{~mm}$ in thickness. There were a total of 265 levels in the reconstruction of the tibial fracture model with internal fixation. The z-axis was constructed according to the real height of the tibia. The junctions were connected in each level and the different levels and structures were distinguished with different colors.
This study was successful in 3D tibial fracture model reconstruction and finite element analysis using Ansys software. However, due to the limited time available for the study, only 3D finite element models of tibias were constructed. In future studies, 3D finite element models of the knee and ankle are required for further investigations.

With regard to the finite element analysis, the results showed that when the mathematical model of tibia was fixed with a load of $1,000 \mathrm{~N}$, there was significant difference in the strain distribution only between the tibia-5 model (14 screws) and tibia-1 model (6 screws fixed at the 1st, 2nd, 3rd, 12nd, 13 th and 14th holes), while there were no significant differences among the tibia-2 (screws at the 1st, 4th, 7th, 8th, 11th and 14th holes), tibia-3 (screws at the 1st, 3rd, 6th, 9th, 12th and 14th holes) and tibia-4 (screws at the 1st, 2nd, 7th, 8th, 13th and 14th holes). When testing the stability against torsion, the strain on the middle of the plate with 14 screws was higher compared with that for 6 screws in tibia-2, -3 and -4 models.

When different fixing positions were used, it was found that the strain of a 1,000- $\mathrm{N}$ axial load was focused on the screws at the center of the plate, with a gradually decreasing trend from top to bottom. These results indicated that with the axial load, the upper screws were easily compressed and broken, which was consistent with the mechanism of clinical LC-DCP plating and screw breakage.

Additionally, it was also found that the strain was the largest when the screws were fixed distal to the fracture (tibia-1 model), which was significantly different compared with the other 6-screw models (tibia-2, tibia-3 and tibia-4 models). Therefore, it is not recommended that the screws are distributed at the ends of the plate in the MIPO treatment of tibial fractures.

For the middle fracture, when 6 screws were used for fixation in the tibia-2, tibia- 3 and tibia- 4 models, the results showed that the tibia- 4 model exhibited the greatest stability against torsion.

The biomechanical results of the present study demonstrated that using a long LC-DPC but fewer screws for fixation of middle tibial fractures had superior biomechanical effects

In conclusion, the use of a 14-hole LC-DCP and with 6 screws for the treatment of middle tibial fractures via MIPO is the most appropriate. The smallest strain per screw is obtained when 2 screws are fixed close to the fracture line (one above and one below the fracture), and a further 4 screws are fixed at the ends of the plate (in the 1st, 2nd, 7th, 8th, 13th and 14th holes).

\section{References}

1. Farouk O, Krettek C, Miclau T, Schandelmaier P, Guy P and Tscherne H: Minimally invasive plate osteosynthesis: does percutaneous plating disrupt femoral blood supply less than the traditional technique? J Orthop Trauma 13: 401-406, 1999.

2. Huang CJ, Zhang YP and Meng SM: Treatment of humerus fractures by closing reduction with locking compression plate through a proximal modified incision. Gu Ke 5: 111-112, 2014 (In Chinese).

3. Tong W, Yang J, Zhu YJ, Xu PL and Shao JK: Minimally invasive percutaneous locking plate osteosynthesis for treatment of osteoporotic humeral fractures in the elderly. Lin Chuang $\mathrm{Gu} \mathrm{Ke} \mathrm{Za}$ Zhi 5: 718-720, 2014 (In Chinese).

4. Feng T: Fixation with intramedullary nail and plate in the treatment of adult humeral shaft fracture:comparison of radial nerve injury and non-union rate. Zhongguo Zu Zhi Gong Cheng Yan Jiu Yu Lin Chuang Kang Fu 29: 2086-2090, 2015 (In Chinese). 
5. Zhang ZZ, Xiong Y and Zhao F: The clinical research of new fixation system for treatment of multi-segmental fractures of humerus. Zhongguo Yi Xue Chuang Xin 27: 135-138, 2014 (In Chinese).

6. Shen J, Yuang TZ, Chen DD, Chang LP and Ma PG: Clinical efficacy of locking compression plate (LCP) with minimally invasive approach in the treatment of humerus thrypsis. Zhongguo Dang Dai Yi Yao 21: 182-183, 2014 (In Chinese).

7. Ma XC, Fu DZ, Lin S, Han YJ, Chen XL,Chen SF, Pu FF and Shao $\mathrm{ZW}$ : Clinical research of the minimally invasive percutaneous plate osteosynthesis for proximal humeral fractures.Shiyong $\mathrm{Gu}$ Ke Za Zhi 20: 102-105, 2014 (In Chinese).

8. Niu XQ, Liu FY and Guo YC: Comparison of three different devices for humeral shaft fracture. Zhonghua Shi Yong Zhen Duan Yu Zhi Liao Zazhi 28: 869-870, 2014 (In Chinese).

9. Huang Y, Xiang M, Hu XC, Chen H, Tang HZ and Yang GY: Application of minimally invasive locking compression plate in treatment of hypertrophic nonunion of humeral shaft fracture for conservative treatment failure. Sichuan Yi Xue 6: 838-841, 2015 (In Chinese).

10. Morgan SJ and Jeray KJ: Minimally invasive plate osteosynthesis in fractures of the tibia. Oper Tech Orthop 11: 195-204, 2001.

11. Gösling T, Schandelmaier P, Marti A, Hufner T, Partenheimer A and Krettek C: Less invasive stabilization of complex tibial plateau fractures: a biomechanical evaluation of a unilateral locked screw plate and double plating. J Orthop Trauma 18: 546-551, 2004.

12. Nobert N, Moremi N, Seni J, Ramesh DM, Ngayomela IH Mshana SE and Gilyoma JM: The effect of early versus delayed surgical debridement on the outcome of open long bone fractures at Bugando Medical Centre, Mwanza, Tanzania. J Trauma Manag Outcomes 10: 6, 2016

13. Hu XJ, Lin BW, Xiao DM, Li WF and Zhang XM: Treatment of tibia shaft fracture by minimally invasive plate osteosynthesis. Shi Yong Yu Fang Yi Xue 13: 734-736, 2006 (In Chinese).

14. Sun YH, Gong WH, Zhu ZN, Chen YQ and Dai R: Treatment of distal tibial fractures using minimally invasive percutaneous plate osteosynthesis. Lin Chuang Gu Ke Za Zhi 7: 9-11, 2004 (In Chinese).

15. Güven M, Unay K, Cakici H, Ozturan EK and Ozkan NK: A new screw fixation technique for minimally invasive percutaneous plate osteosynthesis. Acta Orthop Belg. 74: 846-850, 2008.

16. Lin BW, Xiao DM, Li WF, Wang J, Chen J, Zhang XM, Mai HX, $\mathrm{Lu} \mathrm{XH}$ and Xie WP: Treatment of tibial fracture using minimally invasive percutaneous plate technique. Zhonghua Chuang Shang Za Zhi 18: 551-553, 2002 (In Chinese)

17. Liu CZ, Wu LY, He XY and Wang C: Technology of minimally invasive percutancous plate osteosynthesis for the treatment of fractures of the distal tibia. Zhongguo Gu Shang 21: 213-214, 2008 (In Chinese).
18. Ji F, Tong D, Tang H, Cai X, Zhang Q, Li J and Wang Q: Minimally invasive percutaneous plate osteosynthesis (MIPPO) technique applied in the treatment of humeral shaft distal fractures through a lateral approach. Int Orthop 33: 543-547, 2009.

19. Laurence M, Freeman MAR and Swanson SAV: Engineering considerations in the internal fixation of fractures of the tibial shaft. J Bone Joint Surg Br 51: 754-768, 1969.

20. Rozbruch SR, Müller U, Gautier E and Ganz R: The evolution of femoral shaft plating technique. Clin Orthop 354: 195-208, 1998.

21. Guo SF: The measure of bony structure of lumbar spinal canal and spinal stenosis. Zhonghua Wai Ke Za Zhi 22: 631, 1984 (In Chinese).

22. Qian ZL, Tang TS, Yang HL and Wang YJ: Three-dimension finite element analysis of lumbar intervertebral disc. Suzhou Da Xue Xue Bao 22: 4-7, 2002 (In Chinese).

23. Wang H: Research of a new finite element model of lumbar motion segment. Zhongguo Yi Xue Gong Cheng 13: 185-192, 2005 (In Chinese).

24. Haidukewych GJ: Innovations in locking plate technology. Am Acad Orthop Surg 12: 205-212, 2004.

25. Kozień MS, Lorkowski J, Szczurek S, Hładki W and Trybus M: Computer simulation of the isolated lesion of tibiofibular an syndesmosis using the finite element method. Przegl Lek 65: 50-53, 2008 (In Polish).

26. Field JR, Edmonds-Wilson R and Stanley RM: An evaluation of interface contact profiles in two low contact bone plates. Injury 35: 551-556, 2004.

27. Ellis T, Bourgeault CA and Kyle RF: Screw position affects dynamic compression plate strain in an in vitro fracture model. J Orthop Trauma 15: 333-337, 2001.

28. He JY, Shu Y, Huang SH, Zhou RP, He CJ, Huang J and Pang KJ: The effect of the number of the screw in dynamic compression plates on the stability of plate and screw structure in torsion. Jiangxi Yi Xue Yuan Xue Bao 46: 47-49, 2006 (In Chinese).

29. Liu JC, Yi N, Song XY and Tu ZQ: Causative analysis of fatigue of plates and screws in fractures of shaft of extremital long bone. Hua Xi Yi Xue 15: 187-188, 2000 (In Chinese).

30. Field JR, Törnkvist H, Hearn TC, Sumner-Smith G and Woodside TD: The influence of screws omission on construction stiffness and bone surface strain in the application of bone plate to cadaveric bone. Injury 30: 591-598, 1999.

31. Helfet DL, Shonnard PY, Levine D and Borrelli J Jr: Minimally invasive plate osteosynthesis of distal fractures of the tibia. Injury 28 (Suppl 1): A42-A47, 1997.

32. Baumgaertel F, Buhl M and Rahn BA: Fracture healing in biological plate osteosynthesis. Injury 29 (Suppl 3): C3-C6, 1998. 